\title{
Structures of Poly(vinyl alcohol)/Poly(styrene sulfonic acid) Sodium Salt Blend Hydrogels and Contraction by Absorption of Water into Dried Blend Hydrogels in the Drawn State
}

\author{
Masanobu Nagura, Kohji Usui, Naoya TAKagi, Hiroshi Nishimura, \\ Jyun MuraI, and Yutaka OHKOSHI \\ Faculty of Textile Science and Technology, Shinshu University, \\ Ueda 386, Japan
}

(Received January 22, 1993)

\begin{abstract}
Hydrogels, which have high modulus even with relatively high water content, were prepared by dissolving poly(vinyl alcohol) (PVA) with poly(styrene sulfonic acid) sodium salt (NaPSS) in an autoclave at elevated temperature and pressure, then dehydrating and swelling in water. The high modulus was mainly due to crosslinked structures formed by interpolymer complex not made of PVA crystallites. The contraction of blend gels, which are drawn and dried in the drawn state, is caused by contraction of oriented amorphous chains with absorption of water. The oriented amorphous chains are mainly fixed by the crosslink formed by the interpolymer complex not the PVA crystallites.

KEY WORDS Poly(vinyl alcohol) / Poly(styrene sulfonic acid) Sodium Salt

/ Blend / Hydrogel / Crosslink / Complex / Contraction /
\end{abstract}

It was reported that the physically crosslinked poly(vinyl alcohol) (PVA) hydrogel prepared by repeated freezing and thawing ${ }^{1}$ had high modulus even with relatively high water contents. PVA hydrogels are useful as rubber-like, and highly hygroscopic biomaterials. $^{2,3}$ This high modulus hydrogel contains many immobile water molecules in amorphous regions. ${ }^{4,5}$

Recently rubber-like and physically crosslinked blend hydrogels, which have high modulus even with relatively high water content and have never been known before, were prepared by the following procedure: PVA and another hydrophilic polymer such as anionic poly(sodium L-glutamate) (PSLG) ${ }^{6}$ and nonionic poly(ethylene glycol) $(\mathrm{PEG})^{7}$ were dissolved to prepare a concentrated aqueous solution in an autoclave at elevated temperature and pressure. The very highly viscous coagulate formed was separated, dehydrated and swollen in water. The hydro- gels prepared by blending at normal atmospheric pressure did not have high modulus. We clarified that the relatively high water content caused by a three-dimensional honeycomb structure made by films or tapes in the hydrogels and high modulus was due to crosslinked structures of PVA crystallites and interpolymer complex ${ }^{6,8}$ in the films or tapes.

Tough and rubber-like hydrogels were also prepared from PVA and another anionic poly(styrene sulfonic acid) sodium salt (NaPSS) by same blending procedures as described above, and these hydrogels, drawn and dried in the drawn state, contracted by immersion into water. Amorphous polymers, which are drawn and quenched under the drawn state, contract by heating. ${ }^{9-11}$ However, contraction by immersion in water has never been known before.

In this paper, we investigate the structures and mechanical properties of the rubber-like blend hydrogels of PVA/NaPSS/Water system 
as a function of NaPSS content, and contraction by immersion into water as a function of the molecular orientation of the dried blend gels under the drawn state.

\section{EXPERIMENTAL}

PVA was supplied by KURAREY Co., Ltd., as POVAL-120 (molecular weight 75000, degree of saponification $99.85 \mathrm{~mol} \%$ ). NaPSS (molecular weight 10000) was used an aqueous solution of $20 \mathrm{wt} \%$ supplied by Tohso Ltd. and without further purification.

PVA/NaPSS blend hydrogels were prepared by the following procedure: PVA and NaPSS were dissolved to prepare a $20 \mathrm{wt} \%$ aqueous solution in an autoclave (Taiatsu Glass Kogyou Ltd., TEM-V1000) at 2 atom and $120^{\circ} \mathrm{C}$. The highly viscous coagulate formed was separated, dehydrated and swollen in water. The blend hydrogels obtained were drawn at draw ratios of 2.5 and 3 and dried under the drawn state at room temperature for experiments of contraction and kept in desiccater at a humidity of $33 \%$.

Stress-strain curves were measured using a TENSILON UTM-II-20 at head speed of $10 \mathrm{~mm} \mathrm{~min}^{-1}$. Young's moduli were calculated from the slopes of the stress-strain curves.

DSC experiments were performed on a Seiko Densi instrument DSC-10 at a heating rate of $1^{\circ} \mathrm{C}$ min $^{-1}$ from $-50^{\circ} \mathrm{C}$ after cooling to study the dissolution (melting) of the hydrogels.

Wide angle X-ray scattering experiments were performed using a diffractometer (RIGAKUDENKI 2028) at a scanning rate of $1^{\circ}$ per min. Crystallite orientation percent $(\pi)$ was calculated by eq 1 ,

$$
\pi=(180-\Delta \theta) 100 / 180(\%)
$$

where $\Delta \theta$ is width of half intensity for PVA diffraction peak at $19.6^{\circ}$. Crystallinity was obtained by Hermann's method.

Contraction stress was measured using a TENSILON UTM-II-20.

Contraction percent $(S)$ was calculated by eq
2 ,

$$
\begin{gathered}
S=(\Delta L / L) \times 100(\%) \\
\Delta L=L-L^{\prime}
\end{gathered}
$$

where $L$ and $L^{\prime}$ are lengths of the sample before and after contraction, respectively. The true drawn ratio $\left(\lambda_{\mathrm{s}}\right)$ was calculated by eq 3 ,

$$
\lambda_{\mathrm{s}}=1 /\left(1-S^{\prime}\right)
$$

where $S^{\prime}$ was $S / 100$.

Birefringence $(\Delta n)$ experiments were performed using an Olimpus POM polarized microscope and calculated by eq 4 ,

$$
\Delta n=(N \lambda / d)
$$

where $N, \lambda$, and $d$ are number of streak, wavelength of source light $(0.55 \mu \mathrm{m})$, and thickness of sample, respectively.

\section{RESULTS AND DISCUSSION}

Physical Properties and Structures of PVA/ NaPSS Hydrogels

Figure 1 shows the dependence of Young's modulus of the hydrogel on NaPSS content. The Young's modulus is raised with increasing NaPSS content. Every value is larger than that of PVA hydrogel prepared by repeated freezing and thawing (about $1 \mathrm{MPa}$ ). ${ }^{1}$ Figure 1 also

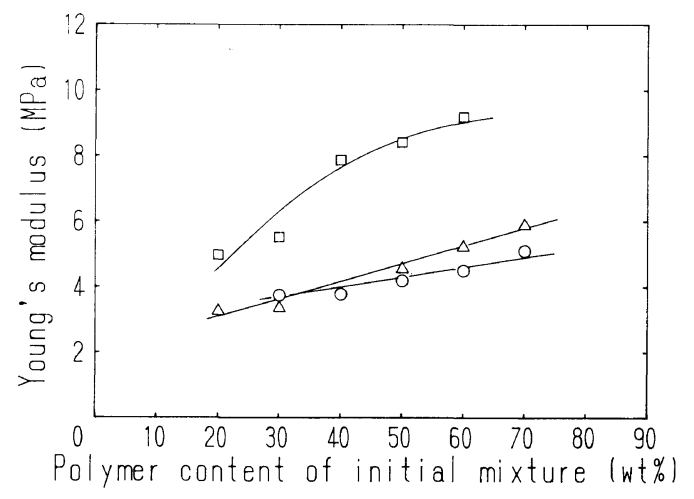

Figure 1. Young's moduli of three blend hydrogel systems consisting of PVA/NaPSS/water ( $-\square-)$, PVA/ PSLG/water $(-\triangle-)$, and PVA/PEG/water (-O-) as a function of the blended polymer content. 
shows the dependence of Young's modulus on the blended polymer content for PVA/PSLG ${ }^{6}$ and PVA $/ \mathrm{PEG}^{7}$ blend hydrogels. Young's moduli of PVA/NaPSS blend hydrogels are higher than these of PVA/PSLG and PVA/ PEG blend hydrogels, because NaPSS has a rigid benzene ring as a side chain and a strongly interacting group such as $\mathrm{SO}_{3} \mathrm{Na}$ group. The increase of Young's modulus suggests that some crosslink points in the hydrogels increase with increasing NaPSS content as discussed below.

The crystallinity of the freeze-dried undrawn hydrogels is shown in Figure 2 as a function of the NaPSS content. The crystallinity is due to only PVA crystallite, because NaPSS is an amorphous polymer, decreasing slightly with increasing NaPSS content in spite of the increase of Young's modulus. Thus the increase of Young's modulus in this study is mainly affected by an another physical crosslink rather than PVA crystallites.

Figure 3 shows the dependence of DSC thermogram of dissolution for the blend hydrogels on NaPSS content. Two broad endothermic peaks appear to overlap. The peak observed at lower temperature (at about $55^{\circ} \mathrm{C}$ ) is caused by the dissolution of the PVA crystallites which act as physical crosslinks in the hydrogels, because PVA crystallites dissolve at similar temperature region in the cases of the PVA gel ${ }^{5}$. This suggests that PVA crystallites grow at a very high rate in the blend hydrogels. There are no peaks in the DSC curve of the concentrated aqueous solution of NaPSS, which does not form gel, while there is a broad peak at higher temperature in the blend hydrogels, which shifts to the higher temperature side with increasing NaPSS content. Thus the peak observed at higher temperature is due to another physically crosslinked structure in the blend hydrogels, which is probably formed by intermolecular hydrogen bonds between hydroxy groups of PVA and strongly interacting $\mathrm{SO}_{3} \mathrm{Na}$ groups of NaPSS as mentioned above. The hydrogen

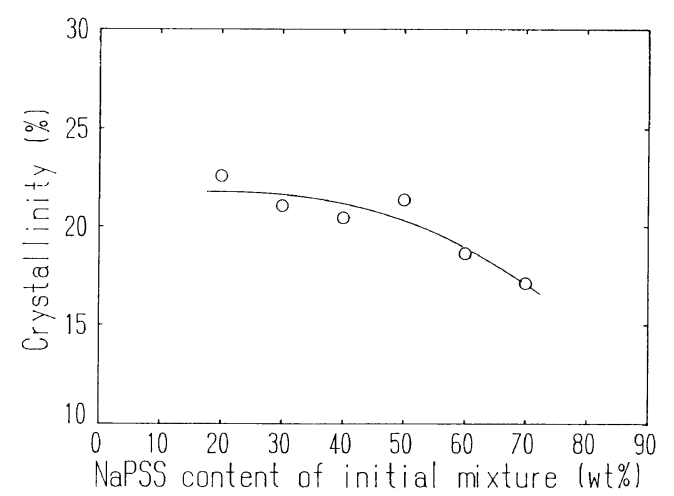

Figure 2. Dependence of crystallinity on NaPSS content.

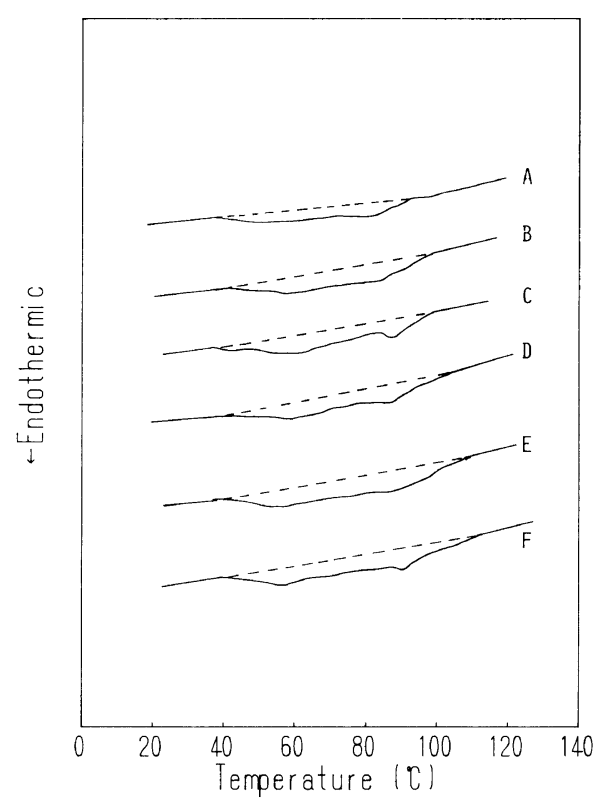

Figure 3. Dependence of DSC thermogram of dissolution for blend hydrogels $\{$ NaPSS content (wt \%): A, 20; B, $30 ; \mathrm{C}, 40 ; \mathrm{D}, 50 ; \mathrm{E}, 60 ; \mathrm{F}, 70\}$.

bonds act like a zipper, and consequently form an interpolymer complex ${ }^{12,13}$ even in concentrated aqueous solution. These results mean that PVA and NaPSS molecules are miscible with each other in the amorphous region of the coagulate, and that the new crosslinked structure by the interpolymer complex also attributes to increase of Young's modulus. These crosslink structures in the blend 


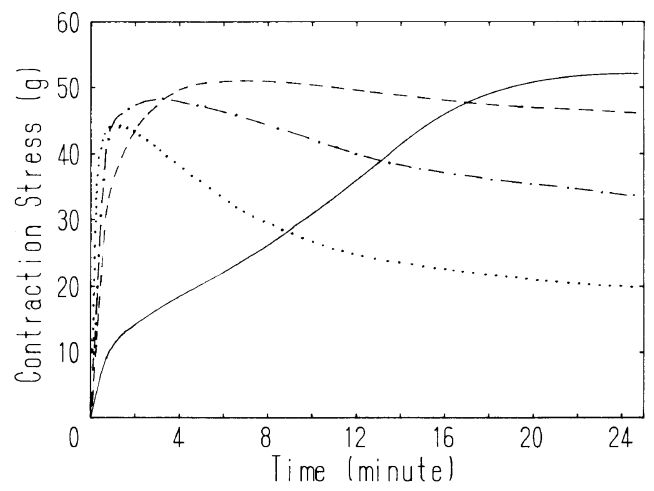

Figure 4. Time dependence of contraction stress in water of blend hydrogels $\{$ PVA $/ \mathrm{NaPSS}=50 / 50(\mathrm{wt} \% / \mathrm{wt} \%)$ and draw ratio of 2$\}$ at $14(-), 30(---), 40(-.-), 50^{\circ} \mathrm{C}$ $(\cdots \cdots)$.

hydrogels were also formed in PVA/PSLG ${ }^{6,8}$ and PVA/PEG blend hydrogels. ${ }^{7}$

\section{Contraction and Molecular Orientation of} Drawn and Dried Blend Hydrogel

Figure 4 shows the time dependence of contraction stress in water of dried blend hydrogels $\{$ PVA/NaPSS $=50 / 50(\mathrm{wt} \% / \mathrm{wt} \%)$, draw ratio $=\times 2\}$ at $14,30,40$, and $50^{\circ} \mathrm{C}$. Maximum contraction stress increases with decreasing temperature and we can see the stress relaxation after the maximum contraction stress above $30^{\circ} \mathrm{C}$. This indicates that the crosslinked structure weakens is destroyed above $30^{\circ} \mathrm{C}$ because of the dissolution of the PVA crystallites as shown in dsc curves (see Figure 3). Thus experiments were performed at $14^{\circ} \mathrm{C}$ to avoid the stress relaxation in following discussion.

Figure 5 shows the dependence of maximum contraction stress in water on the NaPSS content at two draw ratios. The maximum contraction stress increases with increasing NaPSS content and draw ratio. The maximum contraction stress is comparable with that of amorphous engineering plastics drawn and quenched in the drawn state. ${ }^{11-13}$

To study the molecular orientation causing contraction stress, the NaPSS dependence of birefringence at two draw ratios was measured

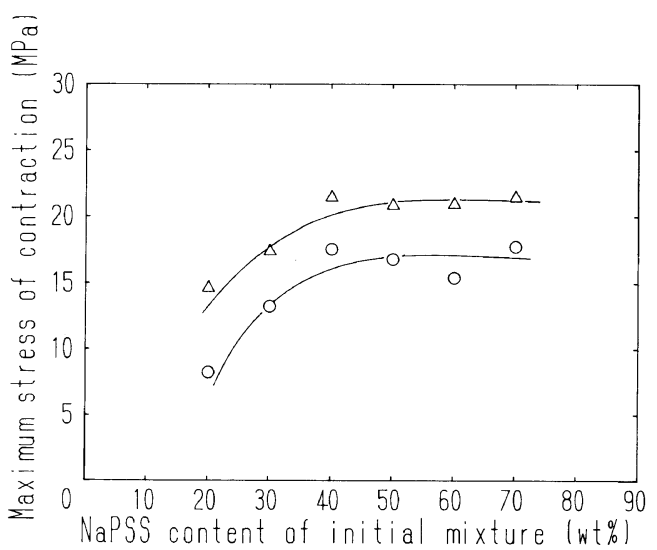

Figure 5. Dependence of maximum contraction stress in water on NaPSS content and draw ratio $(-\mathrm{O}-, \times 2.5$; $-\triangle-, \times 3)$.

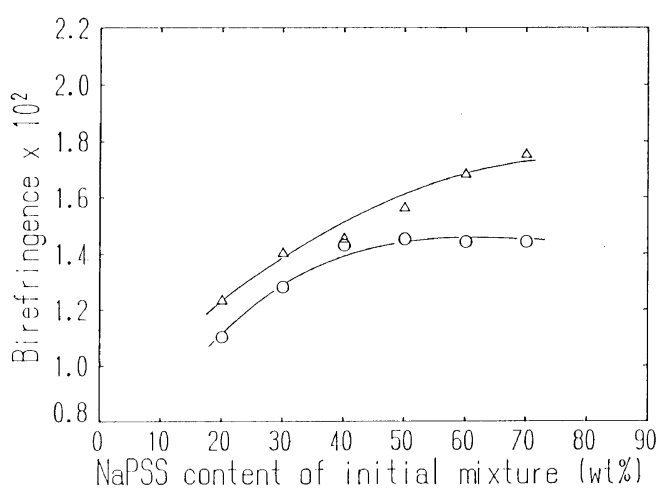

Figure 6. Dependence of birefringence NaPSS content and draw ratio $(-\mathrm{O}-, \times 2.5 ;-\triangle-, \times 3)$.

and the results are shown in Figure 6. The birefringence increases with increasing NaPSS content and draw ratio. Generally birefringence is affected by both orientation of crystallite and of amorphous chain. Thus, the dependence of PVA crystallite orientation on the NaPSS content was elucidated as shown in Figure 7. The PVA crystallite orientation increases with the draw ratio, while the PVA crystallite orientation is constant at every NaPSS content. Since contraction stress increases with NaPSS content at each draw ratio (see Figure 5), contraction with absorption of water is caused by an oriented 


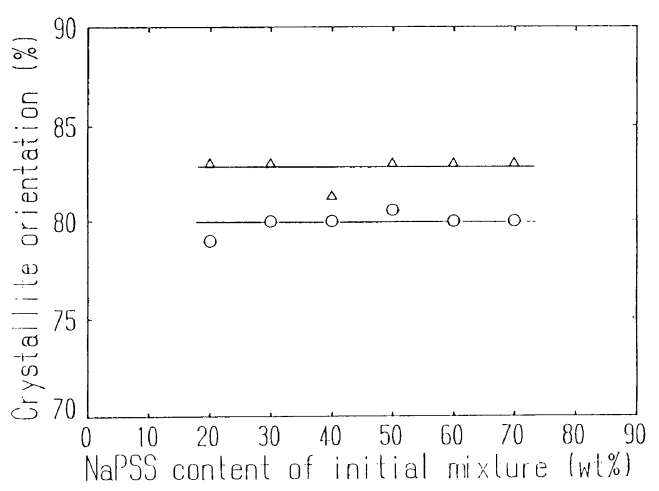

Figure 7. Dependence of PVA crystallite orientation on NaPSS and draw ratio $(-\mathrm{O}-, \times 2.5 ;-\triangle-, \times 3)$.

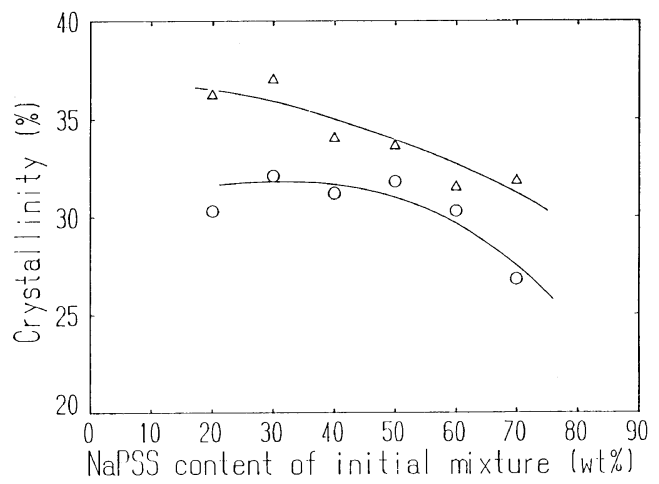

Figure 8. Dependence of PVA crystallinity on NaPSS content and draw ratio $(-\mathrm{O}-, \times 2.5 ;-\triangle-, \times 3)$.

amorphous chain rather than an oriented PVA crystallite. Usually in a drawn crystalline polymer sample, crystallites are rearranged with contraction of the oriented amorphous chain by heating. This means that contraction is not affected by oriented PVA crystallites.

When the hydrogels are drawn, the oriented amorphous chains, which are able to contract with the absorption of water, are fixed probably by the crosslinks formed by crystallites in hydrogel. Figure 8 shows the dependence of PVA crystallinity on NaPSS content and on drawn ratio. The PVA crystallinity decreases with increasing NaPSS content. This means decrease of the fixation effect of the amorphous orientation. From the above speculations and results, we can conclude that oriented amor-

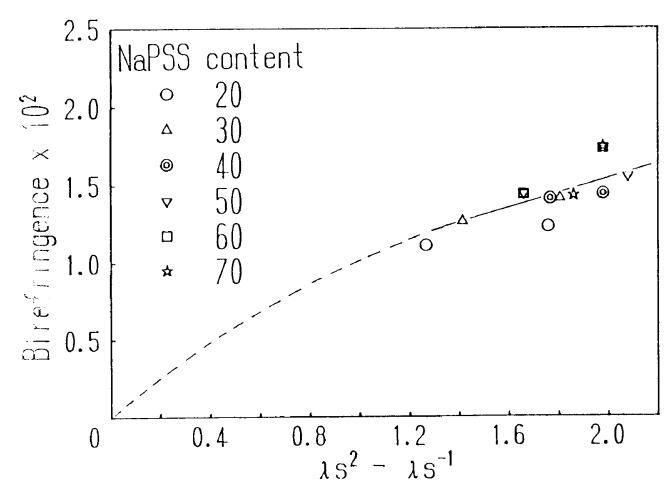

Figure 9. Dependence of birefringence on $\left(\lambda_{\mathrm{s}}{ }^{2}-\lambda_{\mathrm{s}}{ }^{-1}\right)$.

phous chains are mainly fixed by the crosslinks formed by the interpolymer complex and not PVA crystallites.

If contraction is due to an ideal rubber elasticity, birefringence will be proportional to $\left(\lambda_{\mathrm{s}}{ }^{2}-\lambda_{\mathrm{s}}{ }^{-1}\right) .{ }^{14}$ Figure 9 shows the dependence of birefringence on $\left(\lambda_{s}{ }^{2}-\lambda_{s}{ }^{-1}\right)$. Birefringence is not proportional at every draw ratio. Thus, contraction does not show ideal rubber elasticity.

Acknowledgments. The authors thank KURARAY Co., Ltd. for supplying the PVA sample and TOHSO Co. Ltd. for supplying the NaPSS sample.

\section{REFERENCES}

1. M. Nanbu, TRIGGER, 85-8, 83 (1983).

2. M. Nanbu, Kobunshi-Kako, 32, 523 (1983).

3. M. Nanbu, Am. J. Ophthalmol., 99, 494 (1985).

4. M. Nagura, M. Nagura, and H. Ishikawa, Polym. Commun., 25, 313 (1984).

5. M. Nagura, T. Hamano, and H. Ishikawa, Polymer, 30, 762 (1989).

6. M. Nagura, H. Nishimura, and Y. Ohkoshi, Kobunshi Ronbunshu, 48, 517 (1991).

7. M. Nagura, H. Kagawa, T. Maruoka, N. Shiratori, and Y. Ohkoshi, Polymer, in press.

8. M. Nagura and A. Eisenberg, Polymer, 32, 2205 (1991).

9. A. Makinouchi, Sosei to Kako, 20, 618 (1979).

10. T. Machida, N. Okai, and M. Yohda, Sosei to Kako, 25, 45 (1984).

11. M. Karaushi, Plastic Age, 35, 73 (1989). 
12. R. J. Roe and W. C. Zin, Macromolecules, 13, 1221 (1980).

13. Y. C. Bae, S. M. Lambert, D. S. Soane, and J. M.
Prausnitz, Macromolecules, 24, 4403 (1991).

14. N. Saito, "Kobunshi Butsurigaku," Shokabo Press, Tokyo, 1958. 